\title{
Measurement and interpolation uncertainties in rainfall maps from cellular communication networks
}

\author{
M. F. Rios Gaona ${ }^{1}$, A. Overeem ${ }^{1,2}$, H. Leijnse ${ }^{2}$, and R. Uijlenhoet ${ }^{1}$ \\ ${ }^{1}$ Hydrology and Quantitative Water Management Group, Department of Environmental Sciences, Wageningen University, \\ 6708 PB Wageningen, the Netherlands \\ ${ }^{2}$ R\&D Observations and Data Technology, Royal Netherlands Meteorological Institute, 3731 GA De Bilt, the Netherlands \\ Correspondence to: M. F. Rios Gaona (manuel.riosgaona@wur.nl)
}

Received: 24 February 2015 - Published in Hydrol. Earth Syst. Sci. Discuss.: 25 March 2015

Revised: 10 July 2015 - Accepted: 31 July 2015 - Published: 14 August 2015

\begin{abstract}
Accurate measurements of rainfall are important in many hydrological and meteorological applications, for instance, flash-flood early-warning systems, hydraulic structures design, irrigation, weather forecasting, and climate modelling. Whenever possible, link networks measure and store the received power of the electromagnetic signal at regular intervals. The decrease in power can be converted to rainfall intensity, and is largely due to the attenuation by raindrops along the link paths. Such an alternative technique fulfils the continuous effort to obtain measurements of rainfall in time and space at higher resolutions, especially in places where traditional rain gauge networks are scarce or poorly maintained.

Rainfall maps from microwave link networks have recently been introduced at country-wide scales. Despite their potential in rainfall estimation at high spatiotemporal resolutions, the uncertainties present in rainfall maps from link networks are not yet fully comprehended. The aim of this work is to identify and quantify the sources of uncertainty present in interpolated rainfall maps from link rainfall depths. In order to disentangle these sources of uncertainty, we classified them into two categories: (1) those associated with the individual microwave link measurements, i.e. the errors involved in link rainfall retrievals, such as wet antenna attenuation, sampling interval of measurements, wet/dry period classification, dry weather baseline attenuation, quantization of the received power, drop size distribution (DSD), and multi-path propagation; and (2) those associated with mapping, i.e. the combined effect of the interpolation methodology and the spatial density of link measurements.
\end{abstract}

We computed $\sim 3500$ rainfall maps from real and simulated link rainfall depths for 12 days for the land surface of the Netherlands. Simulated link rainfall depths refer to path-averaged rainfall depths obtained from radar data. The $\sim 3500$ real and simulated rainfall maps were compared against quality-controlled gauge-adjusted radar rainfall fields (assumed to be the ground truth). Thus, we were able to not only identify and quantify the sources of uncertainty in such rainfall maps, but also test the actual and optimal performance of one commercial microwave network from one of the cellular providers in the Netherlands. Errors in microwave link measurements were found to be the source that contributes most to the overall uncertainty.

\section{Introduction}

Accurate rainfall estimates are crucial inputs for hydrological models, especially those employed for forecasting flash floods, due to the short timescales in which they develop. Rainfall rates can be retrieved from microwave links because rain droplets attenuate the electromagnetic signal between transmitter and receiver along the microwave link path. The principles behind rainfall estimates from microwave attenuation were investigated by Atlas and Ulbrich (1977). They established the nearly linear relationship between the rainfall intensity and the specific attenuation of the signal for frequencies between 10 and $35 \mathrm{GHz}$.

Messer et al. (2006) and Leijnse et al. (2007) used commercial microwave links to estimate rainfall rates. Note that networks of such links have not been designed for that pur- 
pose. In the last decade several studies have developed methods to improve rainfall estimates from microwave link measurements (Leijnse et al., 2008, 2010; Overeem et al., 2011; Schleiss et al., 2013; Chwala et al., 2014). In addition, Goldshtein et al. (2009) and Zinevich et al. (2008, 2009, 2010) proposed methods to estimate rainfall fields via commercial microwave networks. Giuli et al. (1991) had previously reconstructed rainfall fields from simulated microwave attenuation measurements. Overeem et al. (2011) developed an algorithm to estimate rainfall from minimum and maximum received signal levels over $15 \mathrm{~min}$ intervals, in which the wet antenna effect is corrected for, and where wet and dry spells are identified from the removal of signal losses not related to rainfall by using nearby links.

Rainfall fields can generally be retrieved from commercial microwave link networks at a higher resolution than rain gauge networks. This holds not only for the spatial resolution (usually microwave links outnumber rain gauges) but also for the temporal resolution (microwave link measurements can be obtained for $1 \mathrm{~s}, 1 \mathrm{~min}, 15 \mathrm{~min}$, or daily intervals at either instantaneous or minimum and maximum samples of received signal level (RSL) measurements; Messer et al., 2012). The massive deployment of microwave links provides a complementary network to measure rainfall, especially in countries where rain gauges are scarce or poorly maintained, and where ground-based weather radars are not (yet) deployed (Doumounia et al., 2014).

Recently, Overeem et al. (2013) obtained 15 min and daily rainfall depths from one commercial microwave link network for 12 days for the land surface of the Netherlands $\left(\sim 35000 \mathrm{~km}^{2} ; \sim 1750\right.$ links). They interpolated these rainfall depths to obtain rainfall fields to be compared against gauge-adjusted radar rainfall maps. Although the associated biases were small, the corresponding uncertainties were not. The coefficient of determination, i.e. the square of the correlation coefficient, between link-based and gauge-adjusted radar rainfall maps was 0.49 for the 15 min timescale, and 0.73 for the daily timescale. They did not explore the sources of error that impeded these correlations to reach higher values, though. Here, we address this issue with the aim to unravel and understand the sources of error (and their uncertainties) present in the methodology proposed by Overeem et al. (2013) to estimate rainfall fields. We split the overall uncertainty in rainfall maps from commercial microwave networks into two main sources of error: (1) those associated with the individual microwave link measurements, such as wet antenna attenuation, sampling interval of measurements, wet/dry period classification, dry weather baseline attenuation, drop size distribution (DSD), and multi-path propagation; and (2) those associated with mapping, that is, the combined effect of the interpolation methodology and the spatial density of microwave link measurements. Note that not all the links in the network continuously report data. Only the overall effects of measurement and interpolation errors are addressed here, not all measurement errors separately.
This paper is organized as follows: Sect. 2 describes the data sets and methodology developed by Overeem et al. (2013) to estimate rainfall maps, jointly with the methodologies for this work to derive rainfall maps to identify and quantify error sources. Section 3 compares the results obtained here with those presented in Overeem et al. (2013). Section 4 highlights our major findings. Finally, Sects. 5 and 6 provide a summary, conclusions, and recommendations.

\section{Materials and methods}

\subsection{Data}

Two categories of data were used: link data and radar data. These two data sets are fully independent given that each one originates from a different source: microwave link measurements, and a combination of radar and rain gauge measurements, respectively. Link and radar data contain rainfall depths from the 12-day validation period studied by Overeem et al. (2013), which is spread across the months of June, August, and September 2011. This validation period was selected because of its large number of rainfall events. Figure 1 conceptually illustrates the steps we followed to quantify uncertainties in rainfall maps from link networks.

\subsubsection{Link data}

Link data refer to rainfall depths retrieved from measurements of the attenuation of electromagnetic signals from one commercial microwave link network in the Netherlands. Overeem et al. $(2011,2013)$ thoroughly explained the methodology to convert measurements of the decrease in the received power to rainfall depths, with reference to a level representative of dry weather. Briefly explained, their methodology is based on four steps: (1) a link is considered to be affected by rainfall if the received power jointly decreases with that of nearby links; (2) a reference signal level representative of dry weather, i.e. the median signal level of all dry periods in the previous $24 \mathrm{~h}$, is determined, and the signal is subtracted from this reference level; the result is the attenuation estimate; (3) microwave links for which accumulated (over 1 day) specific attenuation deviates too much (from that of nearby links) are excluded from the analysis; and (4) 15 min average rainfall intensities are computed from a weighted average of minimum and maximum rainfall intensities obtained by a power-law correlation of specific attenuation (Atlas and Ulbrich, 1977). These rainfall intensities are expressed as path-averaged rainfall depths, and are assumed to be representative of the rainfall across the link path. Full details of the algorithm can be found in Overeem et al. (2011, 2013).

Data from up to 1751 link paths are available, with path lengths from 0.13 to $20.26 \mathrm{~km}$, and frequencies from 12.8 to $39.4 \mathrm{GHz}$ (Fig. 2). It is also clear that the network is designed such that the link frequency decreases as path length in- 


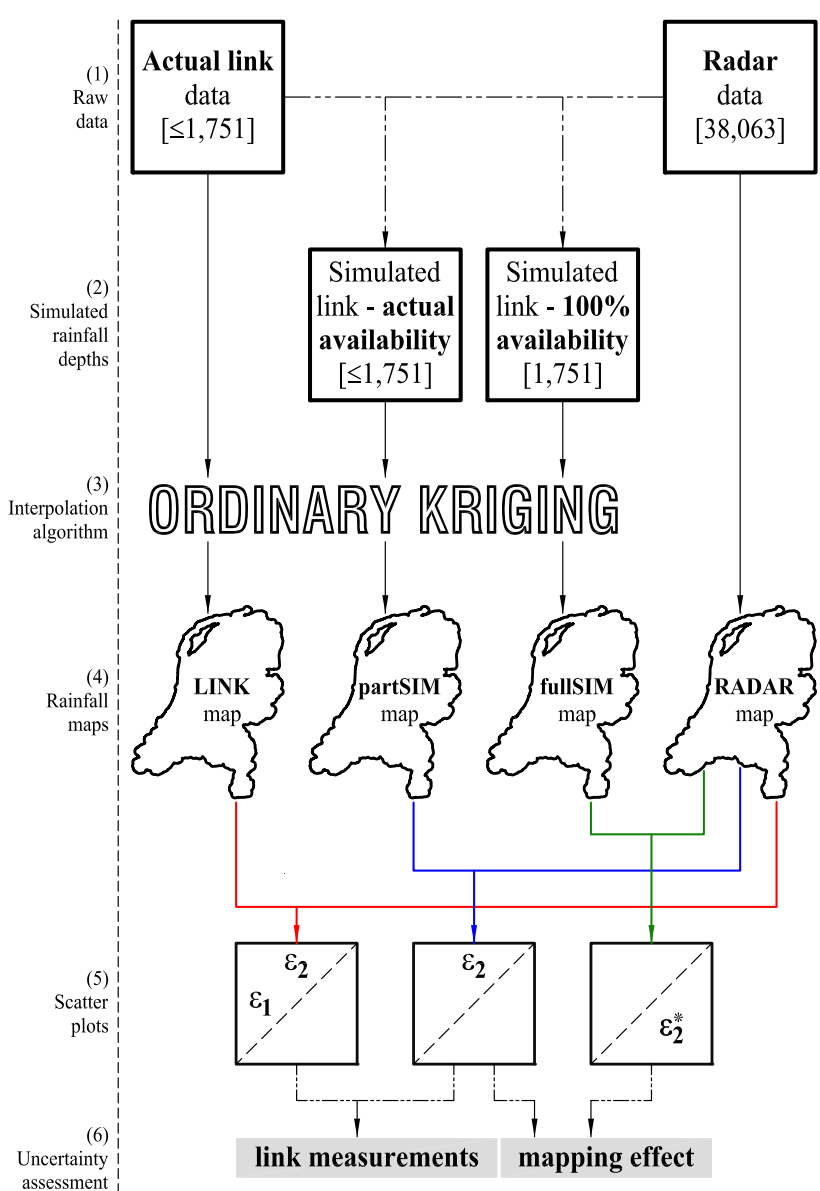

Figure 1. Flowchart to visualize the hierarchical process to identify and quantify uncertainties in rainfall maps from link networks. From top to bottom: (1-2) raw data are selected and rainfall depths simulated; (3-4) through the interpolation methodology rainfall maps are obtained; (5) from the comparison between rainfall maps scatter plots are created; and (6) from the comparison between these scatter plots (and their metrics), the error sources are quantified. $\varepsilon_{1}$ and $\varepsilon_{2}$ represent the categories in which the sources of error are classified. Specifically, $\varepsilon_{1}$ indicates the error from microwave link rainfall retrievals, and $\varepsilon_{2}$ indicates the error related to mapping. $\varepsilon_{2}^{*}$ indicates the best case for the mapping-related error (i.e. all links are available all of the time). The number between brackets (1-2) indicates the number of data for every single map or data set.

creases, mainly because low-frequency links suffer less from rain attenuation.

Figure 3 presents the spatial distribution of one commercial link network from one of the providers in the Netherlands, as well as the temporal availability for each link path. Due to data storage problems, wet/dry classification, and outlier removal, it is not feasible to have link data for all the possible link paths in the network (1751) for every time step. The temporal availability per link varies from 0.9 to $99.9 \%$, with a global average over the entire 12-day data set of $83.5 \%$.

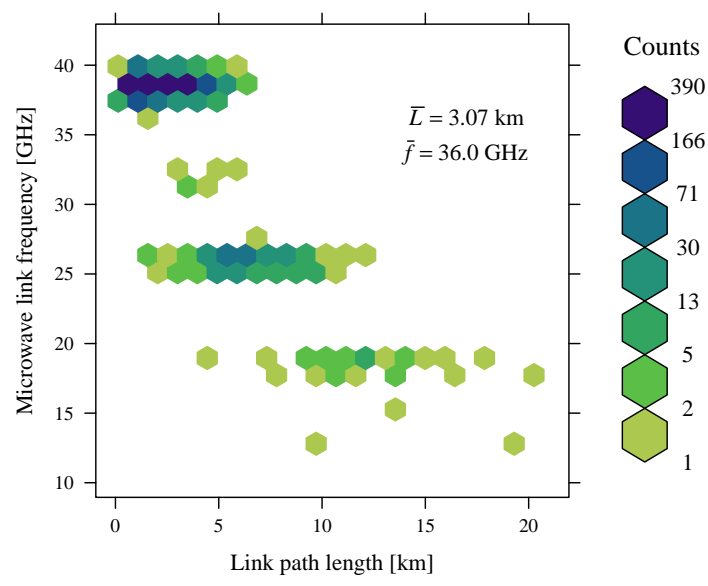

Figure 2. Scatter density plot of microwave link frequencies vs. link-path lengths for the 12-day validation period. The colour scale is logarithmic.

The spatial distribution of the network has two characteristics: (1) there is a strong contrast between urban and rural areas with regard to the spatial distribution of the network; and (2) there are gaps in the network, because of a complete absence of link data or low data availability. Analyses of the link-path orientations show no preferred orientations, i.e. a uniform distribution (such analyses are not presented in this paper).

\subsubsection{Radar data}

Radar data are taken from the climatological rainfall data set $^{1}$ of two C-band Doppler weather radars operated by the Royal Netherlands Meteorological Institute (KNMI) (Overeem et al., 2009a, b, 2011). The composite image of rainfall depths has a temporal resolution of $5 \mathrm{~min}$, and a spatial resolution (pixel size) of $0.92 \mathrm{~km}^{2}$ (rounded to $1 \mathrm{~km}^{2}$ in figures, tables, and subsequent analyses), for the entire land surface of the Netherlands (38063 pixels). This composite image is adjusted with rainfall depths from one automatic and one manual rain gauge network (32 and 325 gauges, respectively) also operated by KNMI. The spatial and temporal resolution, and its accuracy, make this data set a reliable source of rainfall data. We used the same radar data set as that used in Overeem et al. (2013).

\subsection{Simulated link rainfall depths}

Simulated link rainfall depths are averages of radar data based on the topology and time-availability features of the link network. The purpose of simulated link rainfall depths is twofold: (1) to evaluate the performance of the link net-

\footnotetext{
${ }^{1}$ KNMI climatological rainfall data sets are freely available at the IS-ENES climate4impact portal: http: //climate4impact.eu/impactportal/data/catalogbrowser.jsp?catalog= http://opendap.knmi.nl/knmi/thredds/./radarprecipclim.xml.
} 
work assuming that all links provide perfect measurements of path-averaged rainfall at the $15 \mathrm{~min}$ interval, and (2) to evaluate the performance of the link network if all links would be available all the time.

Because link data were obtained in intervals of $15 \mathrm{~min}$, sets of three consecutive 5 min radar composite images were summed up on a pixel-by-pixel basis. The simulation allows us to separate mapping errors from other errors. For detailed studies on the effects of link length and frequency, temporal sampling, power resolution, and wet antenna attenuation in link measurements see Leijnse et al. $(2008,2010)$. After the addition of $5 \mathrm{~min}$ radar composite images, the link network topology was overlaid on the 15 min radar composite image, and all pixels under every link path were selected. Then, for every link path and its associated pixels, rainfall depths were averaged. This was a weighted average in which the weight was taken as the fraction of the total link path that overlaps one radar pixel. For instance, if a $1 \mathrm{~km}$ link path was located $0.6 \mathrm{~km}$ over one pixel and $0.4 \mathrm{~km}$ over a contiguous pixel, the average rainfall depth was the sum of $60 \%$ of the first pixel's rainfall depth plus $40 \%$ of the second pixel's rainfall depth.

Not all link data are available for all the possible link paths in the network (1751) at every time step. In addition to the performance of the actual topology of the network, the complete availability of radar data allowed us to simulate the optimal performance of the link network, i.e. the performance that could theoretically be achieved if all links (1751) would be available all the time.

\subsection{Rainfall maps}

The rainfall depths from actual link measurements and both types of simulations (actual and $100 \%$ network availability) were spatially interpolated to obtain $15 \mathrm{~min}$ rainfall maps with a spatial resolution of $1 \mathrm{~km}^{2}$. In all rainfall maps the land surface of the Netherlands was represented by 38063 pixels. For any given time step, interpolated rainfall maps were compared on a pixel-by-pixel basis against the radar rainfall fields. Hence, $15 \mathrm{~min}$ rainfall maps were obtained for the 12-day validation period, i.e. 1152 rainfall maps in total for each of the four sets of rainfall maps considered (namely, radar, actual links, simulated links with partial availability, and simulated links with $100 \%$ availability). In subsequent figures and tables, these four data sets will be identified as "RADAR", "LINK", "partSIM", and "fullSIM", respectively (see Fig. 1); 15 min rainfall maps were accumulated to daily rainfall maps, i.e. 12 daily rainfall maps per data set.

Ordinary kriging (OK) was employed to generate rainfall maps, because it is the simplest and most straightforward method that accounts for the local variability of the stochastic process, rainfall in this case (Cressie, 1990; Haining et al., 2010). Kriging is ideally suited for interpolation of highly irregularly spaced data points. Nevertheless, this method comes with its own limitations, and a number of assumptions should be made for the method to be valid, e.g.

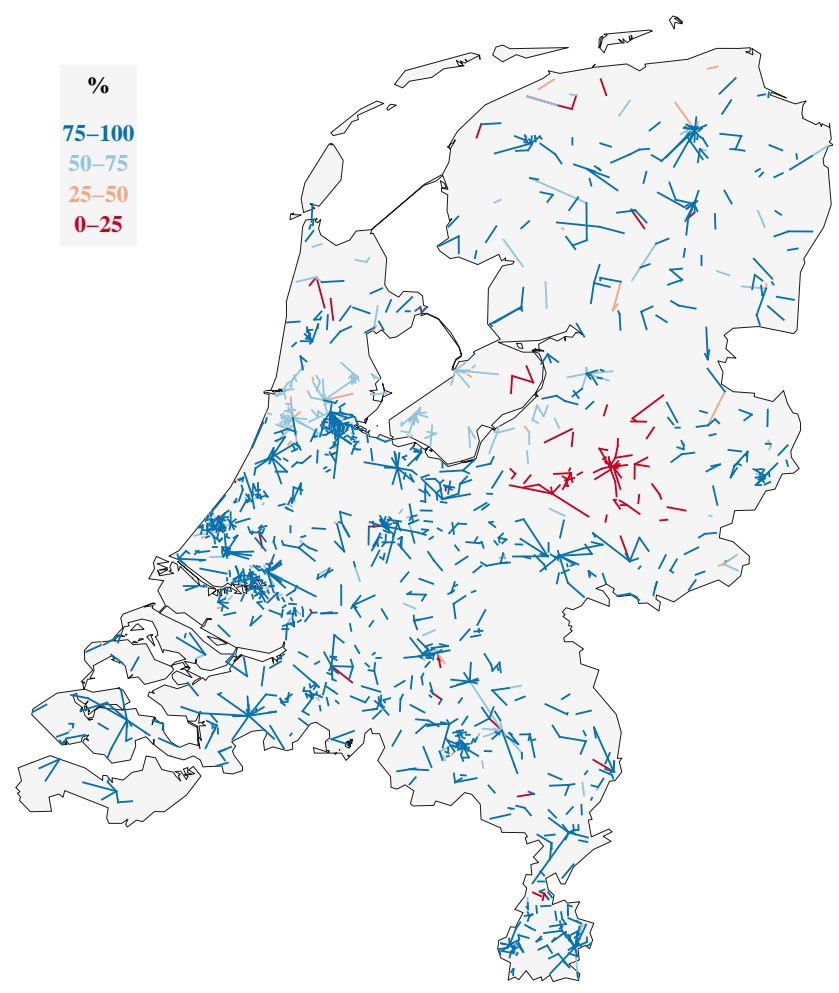

Figure 3. Topology of the T-Mobile NL microwave link network used for this study. The colour scale of the microwave network represents the temporal availability of the link data for the 12-day validation period. The average availability is $83.5 \%$.

isotropy and statistical stationarity. These assumptions are further explained in Sect. 6. The path-averaged link rainfall estimates are assigned to the point at the centre of the link, so that these point data can be used in the OK interpolation. This conversion from line-scale to point-scale data is part of our mapping method, and hence errors resulting from this conversion are part of the mapping uncertainty.

Any kriging method heavily relies on the function that describes the spatial covariance, i.e. the semi-variogram. The semi-variogram is a continuous function that describes how the spatial dependence of a random variable changes with distance and direction (Isaaks and Srivastava, 1989, chap. 7). Like Overeem et al. (2013), we chose the semi-variogram approach of van de Beek et al. (2011) because it is a simple isotropic spherical model developed for the Netherlands on the basis of a 30-year climatological rainfall data set. van de Beek et al. (2011) concluded that the seasonality in range and sill of the semi-variogram can be described by cosine-function models with the day-of-year as the independent variable. Note that they assumed the nugget to be zero. van de Beek et al. (2012) also developed two methodologies that allowed for the spherical semi-variogram to be downscaled from daily to hourly time steps. We chose their second methodology, namely, power-law scaling of cosine-function parameters, because it was shown to perform better. This 
downscaling methodology was based on hourly rainfall data aggregated to $2,3,4,6,8,12$, and $24 \mathrm{~h}$. Here we extended this power-law downscaling to smaller timescales, namely, 0.25 h, i.e. $15 \mathrm{~min}$.

For the LINK, partSIM, and fullSIM data sets, 15 min rainfall maps were obtained as follows: first, the spherical semivariogram parameters were computed and downscaled for the given day of the year. Hence, a single semi-variogram is applied to all $15 \mathrm{~min}$ time steps within that given day. The nugget was defined as $10 \%$ of the sill. Second, rainfall depths were assigned to the coordinates of the link paths' middle points. Third, rainfall depths were interpolated over the spatial grid of the radar data set. The interpolation algorithm always selects the closest 100 rainfall depths to the pixel for which the interpolation is carried out. This selection was established to speed up the interpolation process; $24 \mathrm{~h}$ rainfall maps were obtained from the aggregation of $15 \mathrm{~min}$ rainfall maps.

\subsection{Error and uncertainty metrics}

To quantify the uncertainty in rainfall maps from microwave link networks, we used three metrics: (1) the relative bias, (2) the coefficient of variation, and (3) the coefficient of determination.

The relative bias is a relative measure of the average error between the interpolated and radar rainfall fields (considered to be the ground truth):

relative bias $=\frac{\bar{R}_{\text {res }}}{\bar{R}_{\mathrm{radar}}}=\frac{\sum_{i=1}^{n} R_{\mathrm{res}, i}}{\sum_{i=1}^{n} R_{\mathrm{radar}, i}}$,

where $R_{\mathrm{res}, i}=R_{\mathrm{int}, i}-R_{\mathrm{radar}, i}$. In Eq. (1), $n$ represents all possible pixels and time steps for the 12-day validation period.

The coefficient of variation is a dimensionless measure of dispersion, which is defined as the standard deviation divided by the mean (Haan, 1977). In this case we took the standard deviation of the residuals divided by the mean of the reference field (i.e. the mean of the radar rainfall field):

$\mathrm{CV}=\frac{\sqrt{\frac{1}{n-1} \sum_{i=1}^{n}\left(R_{\mathrm{res}, i}-\bar{R}_{\mathrm{res}}\right)^{2}}}{\bar{R}_{\mathrm{radar}}}$.

The coefficient of variation is a measure of uncertainty (similar to the root mean squared error). For instance, a CV = 0 would indicate a hypothetical case with no bias and no uncertainty, i.e. a case in which all data points would fall exactly on the $1: 1$ line.

The coefficient of determination is a measure of the strength of the linear dependence between two random variables, interpolated and radar rainfall depths, in this case. It is simply defined as the square of the correlation coefficient between the interpolated and radar rainfall depths:

$$
r^{2}=\frac{\left[\sum_{i=1}^{n}\left(R_{\mathrm{radar}, i}-\bar{R}_{\mathrm{radar}}\right) \cdot\left(R_{\mathrm{int}, i}-\bar{R}_{\mathrm{int}}\right)\right]^{2}}{\left[\sum_{i=1}^{n}\left(R_{\mathrm{radar}, i}-\bar{R}_{\mathrm{radar}}\right)^{2}\right] \cdot\left[\sum_{i=1}^{n}\left(R_{\mathrm{int}, i}-\bar{R}_{\mathrm{int}}\right)^{2}\right]} .
$$

The coefficient of determination represents the fraction of the variance of the reference variable that can be explained by a linear regression. In a case of perfect linear correlation, i.e. $r^{2}=1$, all data points would fall on a straight line without any scatter. Hence, the linear regression would be able to explain $100 \%$ of the variance of the reference variable in that case. However, perfect linearity does not imply unbiased estimation because the regression line could not necessarily coincide with the $1: 1$ line, even if it captures all variability.

\section{Results}

From the actual and simulated link rainfall depths, rainfall maps were obtained for three cases: (1) 15 min rainfall maps from interpolation of $15 \mathrm{~min}$ rainfall depths; (2) $24 \mathrm{~h}$ rainfall maps from the sum of these 15 min rainfall maps; and (3) 15 min rainfall maps from interpolation of 15 min rainfall depths, in which each pixel (interpolated rainfall depth) was averaged with the surrounding pixels within a $9 \times 9$ pixel square. The reason for this posterior average of the rainfall depths was to limit representativeness errors in time (Overeem et al., 2013). Incidentally, this area $\left(\sim 81 \mathrm{~km}^{2}\right)$ roughly corresponds to the spatial extent of typical water management units in the Netherlands.

Appendix A presents five examples of $24 \mathrm{~h}$ and $15 \mathrm{~min}$ rainfall maps. Overeem et al. (2013, Supplement) showed daily comparisons between actual link rainfall maps and radar rainfall fields for the 12-day validation period. Here, we present 5 of those 12 cases for reference. These comparisons are extended to both types of simulated link rainfall maps (actual and $100 \%$ network availability) (Fig. A1). Five comparisons of 15 min rainfall maps are also presented (Fig. A2). These examples provide information on the improvement in rainfall fields when the sources of error studied here are removed.

For any given time step, interpolated rainfall maps were compared on a pixel-by-pixel basis against radar rainfall fields. This pixel-by-pixel comparison was done via scatter density plots of interpolated against radar rainfall depths (ground truth). Figure 4 presents an array of scatter plots, for the three cases of spatiotemporal aggregation, for the actual and both types of simulated link rainfall depths (actual and $100 \%$ network availability). Each of the scatter plots in Fig. 4 corresponds to all $15 \mathrm{~min}$ (or $24 \mathrm{~h}$ ) rainfall maps within the 12-day validation period. These plots show paired rainfall 

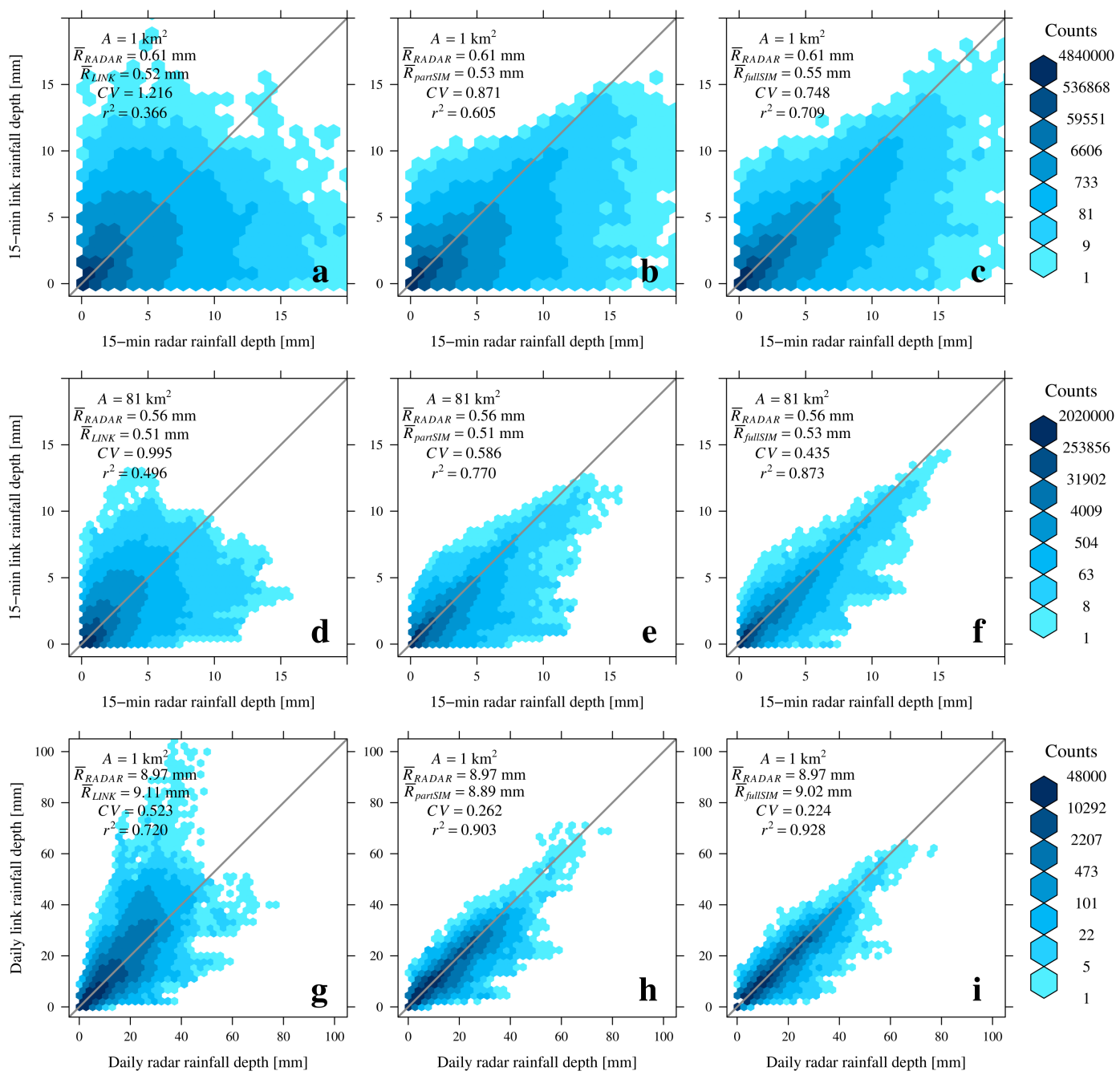

Figure 4. Scatter density plots of interpolated link rainfall depths vs. radar rainfall depths for $15 \mathrm{~min}$ and $24 \mathrm{~h}$. Top row (a, b, c): $15 \mathrm{~min}$ rainfall depths; middle row (d, e, f): 15 min rainfall depths averaged with the surrounding pixels within a $9 \times 9$ pixel square; bottom row $(\mathbf{g}, \mathbf{h}, \mathbf{i})$ : daily sum of 15 min rainfall depths. Left column (a, d, g): actual link rainfall maps vs. radar rainfall fields; centre column (b, e, h): simulated link rainfall maps (actual availability) vs. radar rainfall fields; right column (c, f, i): simulated link rainfall maps (100\% availability) vs. radar rainfall maps. (d) and (g) are comparable to Overeem et al. (2013). The colour scale is logarithmic.

depths of interpolated and radar rainfall maps, for any pair in which the radar rainfall depth is larger than $0.1 \mathrm{~mm}$.

The scatter density plot of Fig. 5 corresponds to the actual and simulated link rainfall depths (actual availability) at the locations of the links, i.e. before any interpolation was applied. Only those pairs for which at least one rainfall depth exceeded $0.1 \mathrm{~mm}$ were plotted.

Table 1 summarizes the values of the relative bias, the coefficient of variation (of the residuals), and the coefficient of determination (i.e. the squared correlation coefficient) for the three cases of spatiotemporal aggregation, for the actual and both types of simulated link rainfall depths.

\section{Discussion}

From left to right and from top to bottom, the general picture that arises from Fig. 4 and Table 1 is (1) a reduced systematic error (relative bias); (2) a smaller random error (CV); and (3) a stronger linear dependence $\left(r^{2}\right)$. This suggests a general improvement of the interpolated link rainfall depths with respect to the radar rainfall depths, as more sources of error are removed from the analysis.

Figure $4 \mathrm{a}, \mathrm{d}$, and g show the relation between the actual link and radar rainfall depths, for the three cases of spatiotemporal aggregation. The scatter in these plots can be attributed to all possible sources of error in rainfall maps from microwave link measurements, i.e. those associated with the 


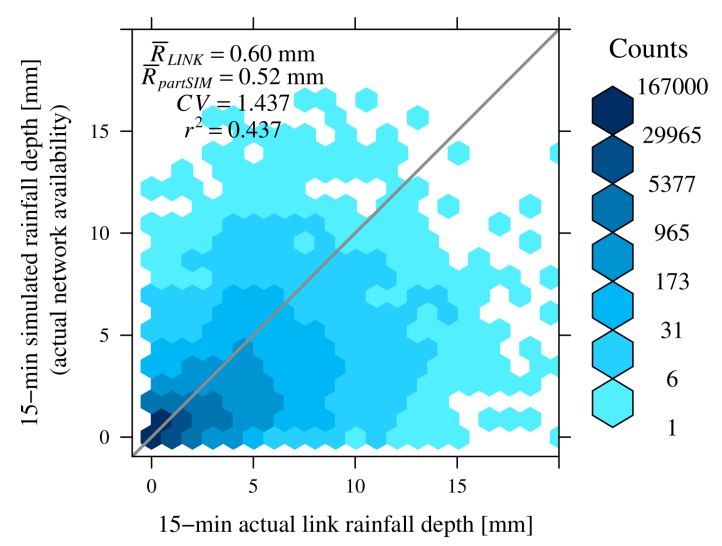

Figure 5. Scatter density plot of simulated link rainfall depths (actual availability) vs. actual link rainfall depths for all 15 min time steps in the 12-day validation period. Both simulated and actual link rainfall depths are path-averaged rainfall depths. The colour scale is logarithmic.

link measurements themselves and those associated with the interpolation of individual measurements (mapping).

The dark blue shading close to the $1: 1$ line for small rainfall depths in all panels of Fig. 4 indicates a good agreement between rainfall estimates from microwave links and radar (note that the colour scale is logarithmic). Conversely, for larger rainfall depths the scatter seems to relatively increase for the actual link measurements (panels a, d, g), while it decreases for the simulated link measurements (all other panels). Such deviations must be the result of errors in individual link measurements as well as the combination of limited spatial coverage of the link network (Fig. 3) with the strong variability of rainfall in space. The relative contribution of the measurement errors to the total error hence increases with rainfall amounts.

From Fig. 4 and Table 1, it is clear as well that the relative bias is most sensitive to the spatial and temporal aggregation level. If all paired rainfall accumulations would have been used (and not only those in which at least the radar rainfall depth exceeds $0.1 \mathrm{~mm}$ ) one would expect the relative bias to be exactly the same for all aggregation levels, because both aggregation and computation of the bias are linear operators (Eq. 1).

There is a limited improvement in terms of the coefficients of variation and determination, when the scatter plots in the second column of Fig. 4 are compared to those in the third column, as well as their respective statistics in Table 1. This means that the main reduction of uncertainty is achieved when the actual link measurements are replaced with the simulated microwave link measurements, rather than to increase the actual link network availability to $100 \%$ for all links. This implies that a significant fraction of the overall uncertainty must be due to errors and uncertainties in the link measurements themselves, rather than due to errors and un-
Table 1. Relative bias, and coefficients of variation and determination for the three cases of spatiotemporal aggregation (15 min [1 km $\left.\left.\mathrm{km}^{2}\right], 15 \mathrm{~min}\left[81 \mathrm{~km}^{2}\right], 24 \mathrm{~h}\left[1 \mathrm{~km}^{2}\right]\right)$, for the three sets of link measurements, i.e. the actual and both types of simulated link rainfall depths (actual and $100 \%$ network availability).

\begin{tabular}{|c|c|c|c|}
\hline & LINK & partSIM & fullSIM \\
\hline \multicolumn{4}{|l|}{ Relative bias [\%] } \\
\hline $15 \min \left[1 \mathrm{~km}^{2}\right]$ & -14.3 & -13.0 & -9.3 \\
\hline $15 \min \left[81 \mathrm{~km}^{2}\right]$ & -9.1 & -9.1 & -5.6 \\
\hline $24 \mathrm{~h}\left[1 \mathrm{~km}^{2}\right]$ & +1.6 & -0.8 & +0.7 \\
\hline \multicolumn{4}{|c|}{ Coefficient of variation - CV } \\
\hline $15 \min \left[1 \mathrm{~km}^{2}\right]$ & 1.216 & 0.871 & 0.748 \\
\hline $15 \min \left[81 \mathrm{~km}^{2}\right]$ & 0.995 & 0.586 & 0.435 \\
\hline $24 \mathrm{~h}\left[1 \mathrm{~km}^{2}\right]$ & 0.523 & 0.262 & 0.224 \\
\hline \multicolumn{4}{|c|}{ Coefficient of determination $-r^{2}$} \\
\hline $15 \min \left[1 \mathrm{~km}^{2}\right]$ & 0.366 & 0.605 & 0.709 \\
\hline $15 \min \left[81 \mathrm{~km}^{2}\right]$ & 0.496 & 0.770 & 0.873 \\
\hline $24 \mathrm{~h}\left[1 \mathrm{~km}^{2}\right]$ & 0.720 & 0.903 & 0.928 \\
\hline
\end{tabular}

certainties associated with mapping, at which rainfall maps are reconstructed.

Figure $4 \mathrm{c}$, $\mathrm{f}$, and $\mathrm{i}$ and the last column of Table 1 indicate the best possible performance that can be achieved with the employed link network (if all links would yield perfect measurements of path-averaged rainfall all the time). The remaining scatter can be attributed to the interpolation methodology (including the assignment of path-averaged rainfall intensities to the link's centre point), the spatial variability of rainfall, and the effect of other factors such as the variable and limited density of the link network (more links in urban than in rural areas).

When 15 min rainfall depths at the $1 \mathrm{~km}^{2}$ spatial scale (Fig. $4 \mathrm{a}-\mathrm{c}$ ) are summed to daily rainfall depths (Fig. $4 \mathrm{~g}-\mathrm{i}$ ), the discrepancies in rainfall estimates at $15 \mathrm{~min}$ tend to cancel each other. This explains the sharp decrease in the coefficient of variation, and the sharp increase in the coefficient of determination between $15 \mathrm{~min}$ and $24 \mathrm{~h}$ rainfall accumulations, which implies a certain degree of independence among the errors in the $15 \mathrm{~min}$ accumulations.

Figure 5 compares simulated against actual link rainfall depths, before any interpolation was applied. This indicates the performance of the 1751 individual links in terms of rainfall retrieval, regardless of the errors and uncertainties introduced by interpolation (mapping). Note that the coefficient of variation is larger than that of the $1 \mathrm{~km}^{2}, 15 \mathrm{~min}$ rainfall accumulations presented in panel a of Fig. 4; and that the coefficient of determination is between those coefficients presented in panels a and d of Fig. 4. If we would assume that rainfall retrieval and mapping errors are independent, we would expect the CV in Fig. 4 to be greater than that in Fig. 5. 

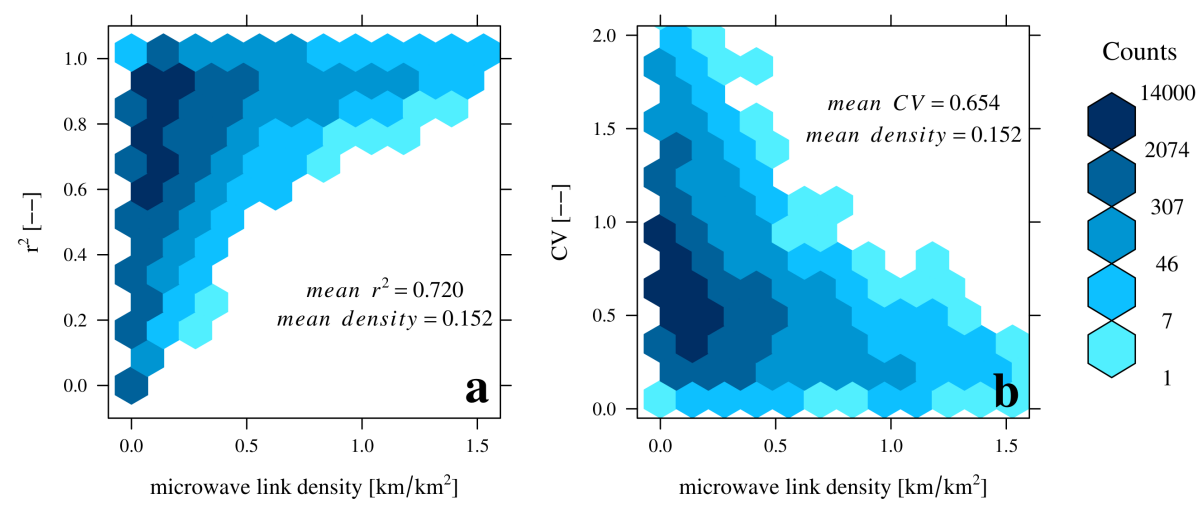

Figure 6. Scatter density plots of coefficient of determination $\left(r^{2}\right)$ and coefficient of variation $(\mathrm{CV})$ vs. microwave link density (averaged over $155 \mathrm{~km}^{2}$ ), for the fullSIM case at $15 \mathrm{~min}$ and $1 \mathrm{~km}^{2}$ spatial scale. The colour scale is logarithmic.

This means that there is a clear interplay between these two types of errors, and that the assumption of independence does not hold. This may be explained by the fact that we use kriging with a variogram that includes a nugget. In areas with a dense link network, the weight of each individual link is relatively small in the computation of the interpolated rainfall field. This reduces the effect of large errors in a given link. In areas with lower link densities the nugget of the employed variogram has a similar reducing effect on large errors.

From Fig. 6 it can be seen that a higher density in the link network guarantees good correlation between the estimated values of rainfall and the ground truth, and a low coefficient of variation of the residuals. From the left panel (Fig. 6a), it can be concluded that lower link densities also contribute (and in large proportion) to higher correlation coefficients. This means that without considering errors in link measurements, these latter being the largest source of uncertainty in country-wide rainfall fields, the network density and the mapping methodology considered here are, respectively, high and good enough to retrieve accurate rainfall fields at such country-wide scales (at least in the Netherlands).

\section{Summary and conclusions}

Our goal was to quantify the errors and uncertainties in rainfall maps from commercial microwave link networks. In general, these errors can be attributed to different sources like wet antenna attenuation, sampling interval of measurements, wet/dry period classification, dry weather baseline attenuation, drop size distribution (DSD), multi-path propagation, interpolation methodology and algorithm, the availability of microwave link measurements, and the variability of rainfall itself across time and space. For the purpose of this paper we classified all possible sources of error into two categories: (1) those associated with the link measurements themselves (retrieval algorithm included), and (2) those associated with mapping. Only the overall effects of physical and interpola- tion errors were addressed here, not all physical errors separately.

To quantify the errors and uncertainties that can be attributed to these two categories, rainfall maps created from three sets of link rainfall depths were compared: actual link measurements, simulated link measurements with the actual network availability, and simulated link measurements with $100 \%$ network availability assumed. Simulated link rainfall depths are not affected by errors and uncertainties attributed to actual link measurements; therefore, we could estimate uncertainties attributed to mapping. Based on a pixel-by-pixel comparison, interpolated rainfall maps of the Netherlands were compared against radar rainfall fields (considered to be the ground truth). These comparisons were carried out on the basis of scatter density plots and three metrics: relative bias, coefficient of variation (CV), and coefficient of determination $\left(r^{2}\right)$.

We found that measurement errors themselves are the source of error that contributes most to the overall uncertainty in rainfall maps from commercial microwave link networks.

In a standard operational framework, data from commercial microwave link networks may not be continuously available for the entire network. Such data gaps affect the accuracy of the retrieved rainfall intensities. Because we were able to simulate rainfall depths on the basis of radar composites, we could investigate the hypothetical case in which data from a commercial link network would be available for all time steps, and for all possible link paths in the network. This best-case scenario could explain an additional $10 \%$ of the variance explained by error-free link measurements with actual network availability for the $15 \mathrm{~min}$ accumulation (3\% for the $24 \mathrm{~h}$ accumulation). Note that these percentages are particular for the region and period considered in this study. Nevertheless, even the best-case scenario showed a remaining and significant amount of uncertainty that could not be removed in rainfall maps. This means that the space-time variability of rainfall is such that it would require an even 
more dense and robust network of microwave links to generate more accurate rainfall maps at country-wide scales. The uncertainties in link rainfall retrievals found in this paper are partly explained by the combined effects of rainfall space variability along the link, non-linearity of the retrieval relation, imperfect temporal sampling strategy, quantization of the received power (data stored in integer number of $\mathrm{dBs}$ ), and wet antenna attenuation (and correction) investigated by Leijnse et al. (2008, in particular Fig. 13, upper right panel on p. 1487). They reported a CV of $\sim 1.0$, which explains a significant part of the CV (1.44) given in Fig. 5. Daily rainfall maps from microwave links showed less uncertainty compared to 15 min rainfall maps, because errors present in 15 min rainfall maps tend to cancel each other when $15 \mathrm{~min}$ rainfall maps are aggregated.

\section{Constraints and recommendations}

The kriging algorithm we used was that of Pebesma (1997) and Pebesma and Wesseling (1998). The interpolated maps from simulated link rainfall depths represent the outcome of a process in which a linear feature (link path) obtained from the average of volume samples (radar data) is assigned to a point (link-path middle point). Each of these features (area, line, volume, point) represents what in geostatistics is referred to as support, i.e. the spatial resolution at which the random variable is analyzed (Cressie and Wikle, 2011, chap 4.1). The arbitrary change from line to point support introduces a source of error that is implicitly included in the errors related to mapping.

Apart from its simplicity and the 30-year rainfall data set on which it is based, we also chose the isotropic spherical semi-variogram of van de Beek et al. (2011), because a consistent semi-variogram model estimated from link data was not feasible for $15 \mathrm{~min}$ rainfall intensities. Isotropic semivariograms assume equal spatial dependence in all possible directions. Rainfall is generally a phenomenon that exhibits anisotropy in time and space (Lepioufle et al., 2012; Velasco-Forero et al., 2009; Guillot and Lebel, 1999; Amani and Lebel, 1997). Nevertheless, it is reasonable to assume isotropy for the Netherlands given its relative small area and flat topography. OK assumes the mean to be constant and unknown within the region of interpolation. When this unknown mean presents substantial changes over short distances, the assumption of statistical stationarity is no longer valid. Universal kriging, kriging with external drift, and regression kriging are more sophisticated interpolation techniques that incorporate trends to account for non-stationarity (e.g. Schuurmans et al., 2007). The performance of these geostatistical techniques to retrieve link rainfall maps was beyond the scope of this research.

If a similar study were to be carried out in a country with different conditions than those present in the Netherlands, three issues should be considered: (1) the spatial and opera- tional configuration of the link network, (2) the climatology of the region where the link network operates, and (3) the spatial scale at which the analysis is carried out.

The first issue, the spatial and operational configuration of the link network, refers to the distribution of link frequencies, lengths, and densities of link networks around the world. For instance, the commercial microwave link network used in this study has an average link-path length of $3.1 \mathrm{~km}$, a mean frequency of $36.0 \mathrm{GHz}$, and a global average availability of $83.5 \%$ across the Netherlands (Figs. 2 and 3). Other regions may have more extensive urban and/or rural areas. In particular, for rural areas one expects to find longer link paths, and therefore lower microwave frequencies. Another issue related to the lower frequencies, e.g. $7 \mathrm{GHz}$, is the low sensitivity to rainfall and the non-linearity of the $R-k$ relationship, mostly in tropical regions (Doumounia et al., 2014). This non-linearity will lead to biases in rainfall intensities in cases of large rainfall variability along the link path (positive biases at lower frequencies where the exponent of the $R-k$ power law is smaller than 1 ; see Leijnse et al., 2010). Thus, the performance of the rainfall retrieval algorithm for such link networks will differ from the performance found in this study. For instance, in places where link paths are longer (tens of kilometres) the error due to spatial variability of rainfall along the link path becomes more important (Berne and Uijlenhoet, 2007; Leijnse et al., 2008, 2010). Moreover, less dense networks with long link paths will provide less detailed information about rainfall.

The second issue, the climatology of the region refers to the local pattern of rainfall that characterizes different regions around the world. The rainfall characteristics of the Netherlands are different from the ones encountered in, e.g., (sub-)tropical regions. For instance, the spherical semivariogram model applied here was derived from climatological rain gauge data for the Netherlands. Furthermore, rainfall characteristics such as raindrop size distributions or the distribution of rainfall intensities will affect the optimal values of the parameters of the retrieval algorithm. Therefore, for regions with different rainfall climatologies than the Netherlands, variations should be considered not only in the interpolation methodology but also in the algorithms and their parameters to retrieve rainfall intensities.

The third issue refers to the spatial scale at which rainfall maps are reconstructed. The analyses presented here focussed on $15 \mathrm{~min}$ (and $24 \mathrm{~h}$ ) maps at 1 and $81 \mathrm{~km}^{2}$, and the differences in error characteristics are significant. For larger regions, for instance, the uncertainty attributed to mapping could play a major role in the overall error distribution. Still, the scale at which rainfall can effectively be retrieved depends greatly on the density of the underlying link network. This means that in regions with a much lower link density than in the Netherlands, the effective spatial resolution for which rainfall maps can be derived will be lower. 


\section{Appendix A: Comparison of $24 \mathrm{~h}$ and $15 \mathrm{~min}$ rainfall maps}

In Fig. A1, the LINK column (top and bottom rows 20110907_08:00 and 20110819_08:00) shows how daily rainfall depths are greatly overestimated by link data, especially in places where there is intense rainfall, and the density of the network is higher. Simulated rainfall depths (actual availability) show improvement of rainfall fields with regard to link-based rainfall fields. Conversely, to actual link rainfall maps, simulated rainfall fields based on the actual availabil- ity of the network present a slight underestimation of rainfall depths. Simulated link rainfall fields (actual and $100 \%$ network availability) are similar because the effect of actual or $100 \%$ availability among $15 \mathrm{~min}$ intervals is smoothed out by the sum of $15 \mathrm{~min}$ rainfall fields.

Figure A2 shows how accurate rainfall events are captured across the Netherlands at 15 min intervals. Note how the accuracy is improved for the best-case scenario of $100 \%$ network availability (fullSIM column). 


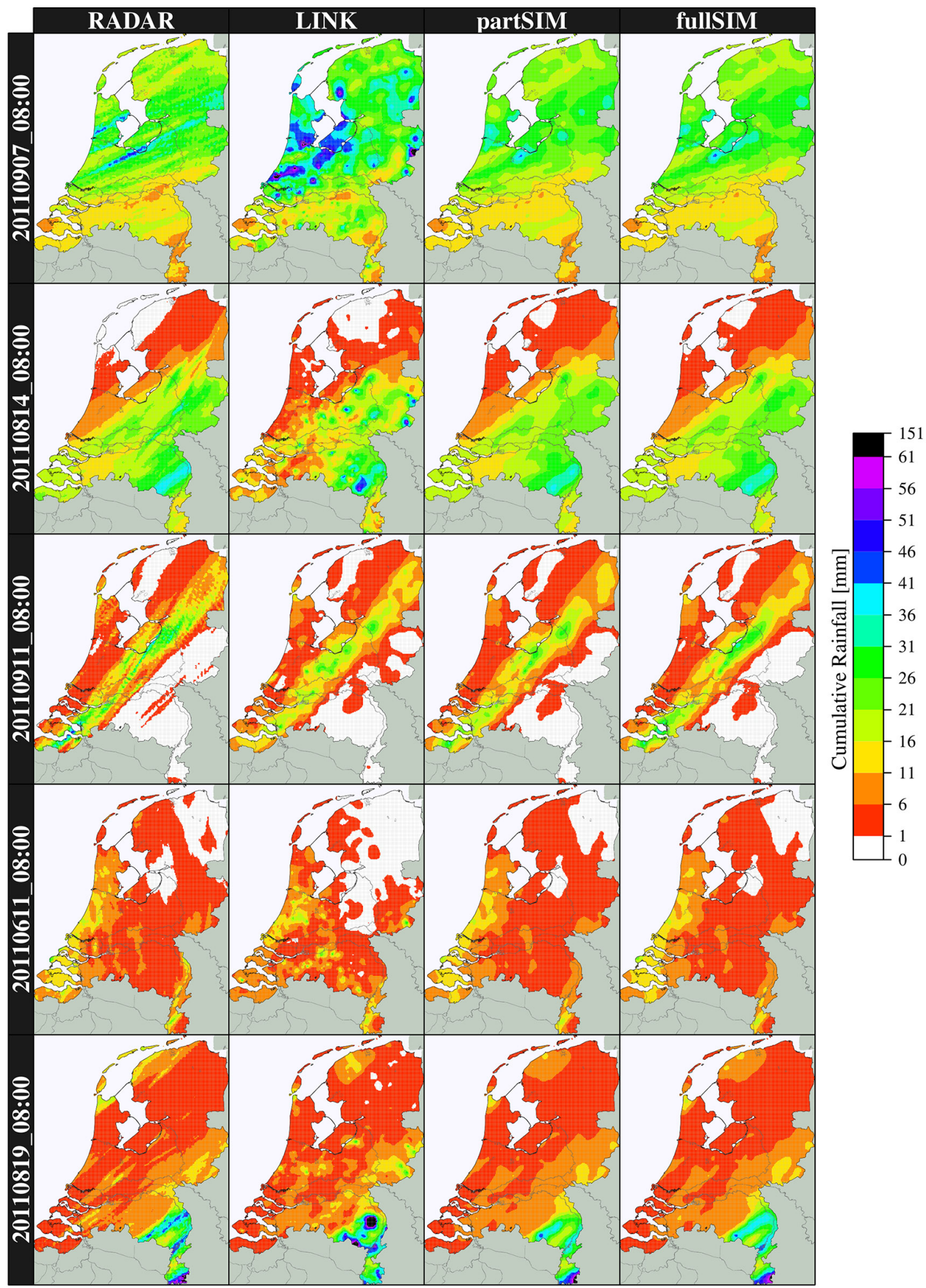

Figure A1. Comparison of daily interpolated rainfall maps with regard to radar rainfall fields (ground truth; left column). The rows show five of the 12 days of the validation period. Daily rainfall maps were aggregated from 15 min rainfall maps. The row labels indicate the end UTC for which the maps were obtained. 


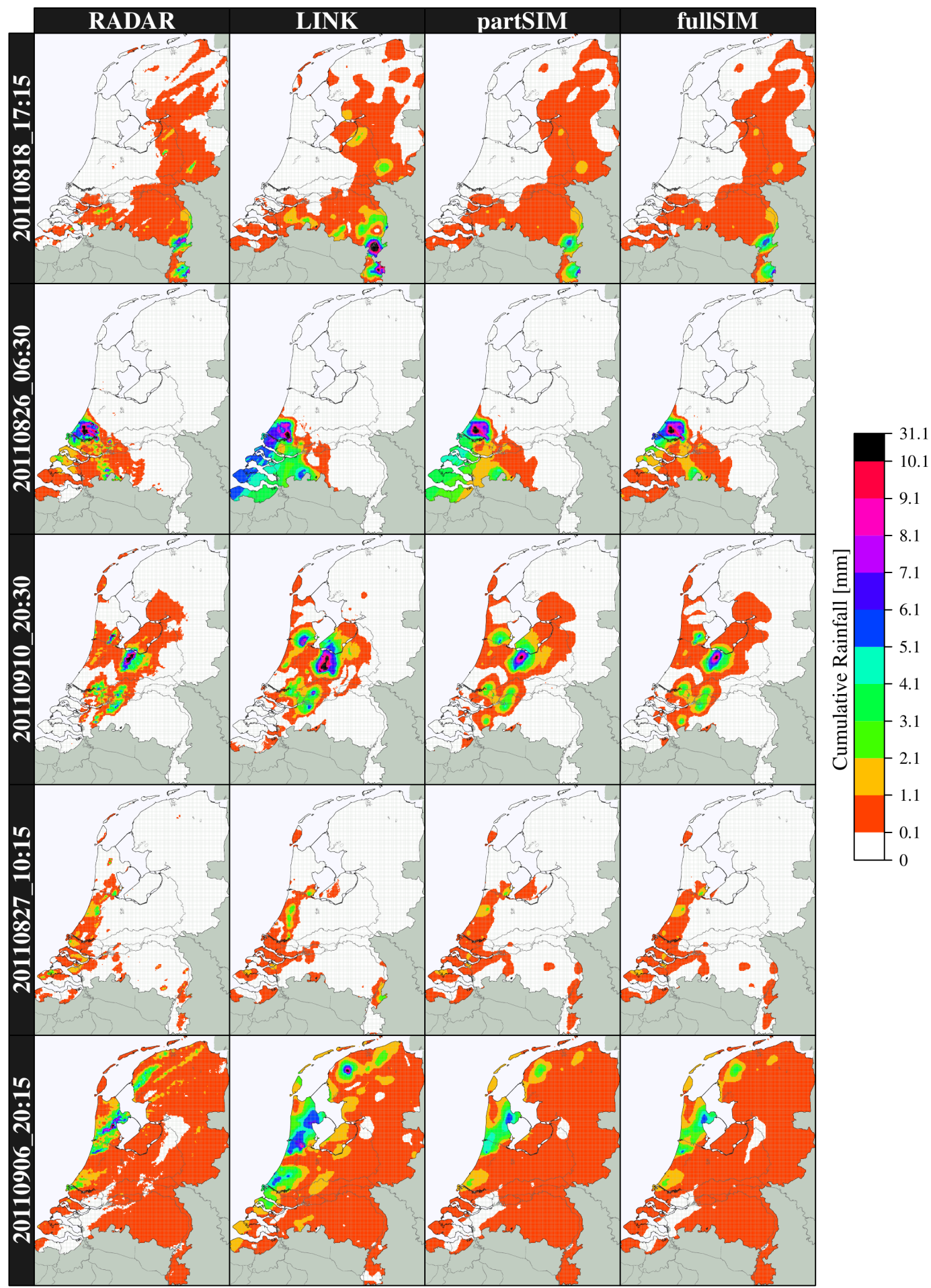

Figure A2. Comparison of $15 \mathrm{~min}$ interpolated rainfall maps with regard to radar rainfall fields (ground truth, left column). The rows show five of the 1152 time steps (cases) present in the 12-day validation period. The row labels indicate the start UTC for which the maps were obtained. 
Acknowledgements. We gratefully acknowledge Ronald Kloeg and Ralph Koppelaar from T-Mobile NL who provided us with the cellular telecommunication link data. We would like to thank Marc Bierkens from Utrecht University for the fruitful discussions. This work was financially supported by the Netherlands Organisation for Scientific Research NWO (project ALW-GO-AO/11-15), and the Netherlands Technology Foundation STW (project 11944).

Edited by: P. Gentine

\section{References}

Amani, A. and Lebel, T.: Lagrangian kriging for the estimation of Sahelian rainfall at small time steps, J. Hydrol., 192, 125-157, doi:10.1016/S0022-1694(96)03104-6, 1997.

Atlas, D. and Ulbrich, C. W.: Path- and area-integrated rainfall measurement by microwave attenuation in the $1-3 \mathrm{~cm}$ band, J. Appl. Meteorol., 16, 1322-1331, doi:10.1175/15200450(1977)016<1322:PAAIRM>2.0.CO;2, 1977.

Berne, A. and Uijlenhoet, R.: Path-averaged rainfall estimation using microwave links: uncertainty due to spatial rainfall variability, Geophys. Res. Lett., 34, L07403, doi:10.1029/2007GL029409, 2007.

Chwala, C., Kunstmann, H., Hipp, S., and Siart, U.: A monostatic microwave transmission experiment for line integrated precipitation and humidity remote sensing, Atmos. Res., 144, 57-72, doi:10.1016/j.atmosres.2013.05.014, 2014.

Cressie, N.: The origins of kriging, Math. Geol., 22, 239-252, doi:10.1007/BF00889887, 1990.

Cressie, N. and Wikle, C. K.: Statistics for Spatio-Temporal Data, Wiley series in Probability and Statistics, Wiley, Oxford, 2011.

Doumounia, A., Gosset, M., Cazenave, F., Kacou, M., and Zougmore, F.: Rainfall monitoring based on microwave links from cellular telecommunication networks: first results from a West African test bed, Geophys. Res. Lett., 41, 6016-6022, doi:10.1002/2014GL060724, 2014.

Giuli, D., Toccafondi, A., Gentili, G. B., and Freni, A.: Tomographic reconstruction of rainfall fields through microwave attenuation measurements, J. Appl. Meteorol., 30, 1323-1340, doi:10.1175/1520-0450(1991)030<1323:TRORFT>2.0.CO;2, 1991.

Goldshtein, O., Messer, H., and Zinevich, A.: Rain rate estimation using measurements from commercial telecommunications links, IEEE T. Signal Proces., 57, 1616-1625, doi:10.1109/TSP.2009.2012554, 2009.

Guillot, G. and Lebel, T.: Approximation of Sahelian rainfall fields with meta-Gaussian random functions, Stoch. Env. Res. Risk. A., 13, 113-130, doi:10.1007/s004770050035, 1999.

Haan, C. T.: Statistical Methods in Hydrology, Iowa State University Press, Ames, 1977.

Haining, R. P., Kerry, R., and Oliver, M. A.: Geography, spatial data analysis, and geostatistics: an overview, Geogr. Anal., 42, 7-31, doi:10.1111/j.1538-4632.2009.00780.x, 2010.

Isaaks, E. H. and Srivastava, R.: Applied Geostatistics, Oxford University Press, available at: http://books.google.nl/books?id= vC2dcXFLI3YC (last access: 23 March 2015), 1989.

Leijnse, H., Uijlenhoet, R., and Stricker, J. N. M.: Hydrometeorological application of a microwave link: 2. Precipitation, Wa- ter Resour. Res., 43, WR004989, doi:10.1029/2006WR004989, 2007.

Leijnse, H., Uijlenhoet, R., and Stricker, J.: Microwave link rainfall estimation: Effects of link length and frequency, temporal sampling, power resolution, and wet antenna attenuation, Adv. Water Resour., 31, 1481-1493, doi:10.1016/j.advwatres.2008.03.004, 2008.

Leijnse, H., Uijlenhoet, R., and Berne, A.: Errors and uncertainties in microwave link rainfall estimation explored using drop size measurements and high-resolution radar data, J. Hydrometeorol., 11, 1330-1344, doi:10.1175/2010JHM1243.1, 2010.

Lepioufle, J.-M., Leblois, E., and Creutin, J.-D.: Variography of rainfall accumulation in presence of advection, J. Hydrol., 464465, 494-504, doi:10.1016/j.jhydrol.2012.07.041, 2012.

Messer, H., Zinevich, A., and Alpert, P.: Environmental monitoring by wireless communication networks, Science, 312, p. 713, doi:10.1126/science.1120034, 2006.

Messer, H., Zinevich, A., and Alpert, P.: Environmental sensor networks using existing wireless communication systems for rainfall and wind velocity measurements, IEEE Instru. Meas. Mag., 15, 32-38, doi:10.1109/MIM.2012.6174577, 2012.

Overeem, A., Buishand, T. A., and Holleman, I.: Extreme rainfall analysis and estimation of depth-duration-frequency curves using weather radar, Water Resour. Res., 45, WR007869, doi:10.1029/2009WR007869, 2009a.

Overeem, A., Holleman, I., and Buishand, A.: Derivation of a 10year radar-based climatology of rainfall, J. Appl. Meteorol. Clim., 48, 1448-1463, doi:10.1175/2009JAMC1954.1, 2009 b.

Overeem, A., Leijnse, H., and Uijlenhoet, R.: Measuring urban rainfall using microwave links from commercial cellular communication networks, Water Resour. Res., 47, WR010350, doi:10.1029/2010WR010350, 2011.

Overeem, A., Leijnse, H., and Uijlenhoet, R.: Country-wide rainfall maps from cellular communication networks, P. Natl. Acad. Sci. USA, 110, 2741-2745, doi:10.1073/pnas.1217961110, 2013.

Pebesma, E. J.: Gstat user's manual, available at: http://www.gstat. org/gstat.pdf (last access: 23 March 2015), 1997.

Pebesma, E. J. and Wesseling, C. G.: Gstat: a program for geostatistical modelling, prediction and simulation, Comput. Geosci., 24, 17-31, doi:10.1016/S0098-3004(97)00082-4, 1998.

Schleiss, M., Rieckermann, J., and Berne, A.: Quantification and modeling of wet-antenna attenuation for commercial microwave links, IEEE Geosci. Remote S., 10, 1195-1199, doi:10.1109/LGRS.2012.2236074, 2013.

Schuurmans, J. M., Bierkens, M. F. P., Pebesma, E. J., and Uijlenhoet, R.: Automatic Prediction of High-Resolution Daily Rainfall Fields for Multiple Extents: The Potential of Operational Radar, J. Hydrometeorol., 8, 1204-1224, doi:10.1175/2007JHM792.1, 2007.

van de Beek, C. Z., Leijnse, H., Torfs, P. J. J. F., and Uijlenhoet, R.: Climatology of daily rainfall semi-variance in The Netherlands, Hydrol. Earth Syst. Sci., 15, 171-183, doi:10.5194/hess-15-1712011, 2011.

van de Beek, C. Z., Leijnse, H., Torfs, P., and Uijlenhoet, R.: Seasonal semi-variance of Dutch rainfall at hourly to daily scales, Adv. Water Resour., 45, 76-85, doi:10.1016/j.advwatres.2012.03.023, 2012.

Velasco-Forero, C. A., Sempere-Torres, D., Cassiraga, E. F., and Gómez-Hernández, J. J.: A non-parametric automatic 
blending methodology to estimate rainfall fields from rain gauge and radar data, Adv. Water Resour., 32, 986-1002, doi:10.1016/j.advwatres.2008.10.004, 2009.

Zinevich, A., Alpert, P., and Messer, H.: Estimation of rainfall fields using commercial microwave communication networks of variable density, Adv. Water Resour., 31, 1470-1480, doi:10.1016/j.advwatres.2008.03.003, 2008.
Zinevich, A., Messer, H., and Alpert, P.: Frontal rainfall observation by a commercial microwave communication network, J. Appl. Meteorol. Clim., 48, 1317-1334, doi:10.1175/2008JAMC2014.1, 2009.

Zinevich, A., Messer, H., and Alpert, P.: Prediction of rainfall intensity measurement errors using commercial microwave communication links, Atmos. Meas. Tech., 3, 1385-1402, doi:10.5194/amt-3-1385-2010, 2010. 